\title{
Intravascular Lymphomatosis Mimicking Primary Central Nervous System Lymphoma: A Case Report and Literature Review
}

\author{
Masahiro Oomura $^{a} \quad$ Noriyuki Sakakibara $^{b}$ Shugo Suzuki ${ }^{c}$ \\ Atsushi Wakita $^{d}$ Yuji Mori $^{\mathrm{e}}$ Kaoru Kamimoto $^{a}$ \\ Departments of a Neurology, ${ }^{b}$ Dermatology, ${ }^{\mathrm{C} P a t h o l o g y,}{ }^{\mathrm{d}} \mathrm{Hematology}$ and ${ }^{\mathrm{e}}$ Radiology, \\ Nagoya City East Medical Center, Nagoya, Japan
}

\section{Key Words}

Intravascular large B-cell lymphoma - Fever of unknown origin - Random skin biopsy · Brain mass · Diffusion-weighted image

\section{Abstract}

We herein report a 75-year-old female patient with intravascular lymphomatosis (IVL) who presented with fever of unknown origin. Examination, including contrast-enhanced CT and ${ }^{67} \mathrm{Ga}$ scintigraphy, failed to show any lesions. Her blood levels of lactate dehydrogenase and soluble interleukin-2 receptors were high, suggesting a lymphomatous tumor. A bone marrow puncture was negative, and a random skin biopsy revealed a monoclonal proliferation of naked, large lymphocytes in the vascular space of the subcutaneous tissue, confirming the diagnosis of IVL. MRI, performed 7 weeks after admission, showed a brain mass mimicking primary central nervous system lymphoma. The mass was considered to be a collection of malignant lymphocyte cells invading from the vessels. Without the random skin biopsy, this case may have been misdiagnosed as primary central nervous system lymphoma.

(c) 2014 S. Karger AG, Basel

\section{Introduction}

Intravascular lymphomatosis (IVL) is an uncommon systemic disease in which malignant proliferation of lymphocytes occurs exclusively in the vessels [1]. The clinical symptoms are nonspecific in most cases, making an early diagnosis difficult [1, 2]. A unique

Masahiro Oomura, MD

Department of Neurology

Nagoya City East Medical Center

1-2-23 Wakamizu, Chikusa-ku, Nagoya, Aichi 464-8547 (Japan)

E-Mailm.oomura@gmail.com 
Oomura et al.: Intravascular Lymphomatosis Mimicking Primary Central Nervous System Lymphoma: A Case Report and Literature Review

characteristic of this disorder is that the proliferation of malignant lymphocytes occurs only within the vessels, in contrast to primary central nervous system lymphoma (PCNSL). We herein report a patient with IVL who presented with fever of unknown origin (FUO) and in whom a brain mass developed, mimicking PCNSL. A random skin biopsy confirmed the diagnosis of IVL.

\section{Case Report}

A 75-year-old right-handed female patient was admitted to our hospital for the evaluation of recurrent fevers. She had been well until 3 weeks before admission, when fever and fatigue developed. She had a history of cholecystectomy, spinal compression fracture, and an old cerebral infarction of the left middle cerebral artery. After the brain infarction, she developed aphasia and right hemiplegia and became dependent on a wheelchair. She showed paroxysmal atrial fibrillation, and warfarin was prescribed to prevent a second stroke.

On examination, her blood pressure was $150 / 70 \mathrm{~mm} \mathrm{Hg}$, her pulse 90 beats per minute, and her body temperature was $37.5^{\circ} \mathrm{C}$. There was a small decubitus ulcer on the sacrum and a nonpitting edema in both legs. No lymph node swelling was noted. Neurological examination revealed aphasia and right hemiplegia. The rest of the examination was normal. Her white blood cell count was $11,000 / \mathrm{mm}^{3}$ (reference range $3,500-8,000 / \mathrm{mm}^{3}$ ). Her blood levels of lactate dehydrogenase (LDH), C-reactive protein, and soluble interleukin-2 receptor (sIL-2R) were high at $458 \mathrm{IU} / \mathrm{l}$ (normal $<229 \mathrm{IU} / \mathrm{l}$ ), $16.8 \mathrm{mg} / \mathrm{dl}$ (normal $<0.8 \mathrm{mg} / \mathrm{dl}$ ), and $3,458 \mathrm{U} / \mathrm{ml}$ (normal $<515 \mathrm{U} / \mathrm{ml}$ ), respectively. Her blood levels of electrolytes, glucose, alkaline phosphatase, alanine aminotransferase, aspartate aminotransferase, amylase, brain natriuretic protein, and vitamin B1 were normal, as were her renal and thyroid function. Lumbar puncture was performed; there was no pleocytosis in the cerebrospinal fluid, but the protein and IgG levels were increased to $85 \mathrm{mg} / \mathrm{dl}$ (normal $<50 \mathrm{mg} / \mathrm{dl}$ ) and $17 \mathrm{mg} / \mathrm{dl}$ (normal $<4 \mathrm{mg} / \mathrm{dl}$ ), respectively. A urine culture showed no growth. Blood specimens were sent for cultures of bacteria, mycobacterium, and fungi. All results were negative. A contrastenhanced CT of the chest, abdomen, and pelvis failed to reveal any abnormal lesions that could have caused the fever. No adrenal gland mass was noted. A brain MRI obtained 3 weeks after admission showed an old infarction but was otherwise normal. Tests for antibodies to hepatitis B, hepatitis C, human immunodeficiency virus, human Tlymphotropic virus, and the Treponema pallidum were negative. Antibiotic treatments including ciprofloxacin at $600 \mathrm{mg} /$ day, panipenem/betamipron at $1 \mathrm{~g} /$ day, and ceftazidime at $2 \mathrm{~g} /$ day combined with arbekacin at $200 \mathrm{mg}$ /day were undertaken, but all were ineffective. ${ }^{67} \mathrm{Ga}$ scintigraphy showed no abnormal uptake. The fever continued for more than 3 weeks and fulfilled the criteria for FUO. Because the serum levels of LDH and sIL-2R remained high, we suspected a hematogenous tumor such as myeloma, and bone marrow puncture was performed, but the result was normal. Although there were no skin lesions, we strongly suspected IVL and thus performed a random skin biopsy. The biopsy was obtained from 1 lesion of the purpura on the abdomen and 2 regions of the skin with a normal appearance on the abdomen and left thigh. Histological examination revealed atypical cells lodged in the intravascular lumens in the subcutaneous tissue of the normally appearing left thigh region (fig. 1a). Higher magnification revealed vessels filled with large malignant lymphoid cells and eosinophilic nucleoli (fig. 1b); the cells were stained with CD20 antibodies but not with CD3 (fig. 1c). The patient was thus diagnosed with IVL (intravascular large B-cell lymphoma). Her consciousness deteriorated 6 weeks after admission. An MRI 
Oomura et al.: Intravascular Lymphomatosis Mimicking Primary Central Nervous System Lymphoma: A Case Report and Literature Review

obtained 7 weeks after admission showed a mass lesion involving the right basal ganglia, thalamus, corona radiata, uncus, and crus cerebri (fig. 2). The mass was isointense on a T1weighted image, hyperintense on a T2-weighted image, and exhibited mild restricted diffusion on a diffusion-weighted image (DWI). The mass was homogeneously enhanced (fig. 2). No definite ischemic lesions were noted. All radiological findings were compatible with those of PCNSL. The patient's status was too poor for chemotherapy. An MRI obtained 9 weeks after admission showed an enlargement of the mass (fig. 2). She died 11 weeks after admission. Permission for a postmortem examination was not obtained.

\section{Discussion}

IVL is an uncommon systemic disease in which malignant proliferation of lymphocytes occurs exclusively in vessels. The clinical presentation of IVL is frequently nonspecific and antemortem diagnosis is difficult [1]. DiGiuseppe et al. [2] studied 10 cases of IVL in a single institution and reported that FUO and a mental status change were the most common signs, being present in $60 \%$ of cases. They reported that the duration of FUO ranged from 2 to 6 months [2]. In our patient, FUO had been present for 7 weeks until the diagnosis of IVL was made. In patients with long-standing FUO, IVL should be included as a differential diagnosis.

Histological confirmation of the malignant proliferation of lymphocytes in vessels is required for the diagnosis of IVL. Although the number of reported cases of IVL in which antemortem diagnosis was achieved by biopsy is small, such biopsies reportedly involved the brain, adrenal gland, kidney, liver, spleen, lung, bone marrow, muscles, and the skin [3]. Of these organs, the skin is the most accessible. Because dermatological manifestations including nodules, plaques, and macules are present in up to one third of patients with IVL [1], skin biopsies should be conducted when any skin lesions are found in patients in whom IVL is suspected. Asada et al. [4] recently reported the usefulness of random skin biopsies for the early diagnosis of IVL. He reported 6 patients with IVL who were diagnosed by random skin biopsies [4]. Skin lesions were noted in only 2 out of these 6 patients [4]. The involvement of clinically unaffected skin has been reported in autopsy findings, and this involvement serves as the basis for a random skin biopsy. In our case, no definite skin lesion suggestive of IVL was noted during the clinical course. We performed a random skin biopsy because of the strong suspicion of IVL based on the high serum levels of LDH and sIL-2R. This led to the definitive diagnosis of IVL.

The MRI findings of IVL have not been well characterized because the number of reported cases is relatively small. However, the most commonly reported MRI findings are multifocal lesions that are hyperintense on T2-weighted images, mimicking small infarctions. Baehring et al. [5] recently evaluated 5 patients with IVL in whom serial diffusionweighted MRIs were obtained. They reported that multifocal DWI lesions are the most common observations, suggesting the presence of small-vessel ischemia that is probably caused by the occlusion of vessels by malignant lymphomatous cells [5]. A brain mass developed in the present patient. The mass was isointense on T1-weighted images and hyperintense on T2-weighted images and it showed a restricted diffusion on DWI and homogeneous Gd enhancement. The differential diagnoses for a brain lesion appearing hyperintense on T2-weighted images with Gd enhancement may include PCNSL, toxoplasmosis, glioblastoma multiforme, abscess, metastasis, tumefactive multiple sclerosis, and primary angiitis of the central nervous system (PACNS). Of these etiologies, homogeneous Gd enhancement and restricted diffusion on DWI make PCNSL most likely, and the serological findings suggesting a lymphomatous disease could have allowed for a diagnosis 
Oomura et al.: Intravascular Lymphomatosis Mimicking Primary Central Nervous System Lymphoma: A Case Report and Literature Review

of PCNSL in the present case if the brain biopsy had not been performed. Moreover, when the mass appeared, there were no definitive ischemic lesions, which are common radiological findings of IVL. Brain abscess is an important etiology in patients with FUO. Antibiotic treatment was not effective and blood culture was negative in this case; however, there was still a probability of developing a brain abscess after admission. Despite this, the lack of a characteristic thick rim of contrast enhancement around the lesion was considered to make a brain abscess unlikely. PACNS is a rare vasculitis of unknown etiology that exclusively involves the central nervous system. Commonly reported MRI findings of PACNS are multifocal deep gray and subcortical hyperintensities on T2-weighted images. Recently, some cases of PACNS have been reported to present as brain masses, mimicking brain tumors [6]. Similar to IVL, PACNS can present with a wide variety of clinical symptoms. The low sensitivity of the characteristic angiographic signs and the lack of specific findings in general medical laboratory investigations make a brain biopsy necessary for the diagnosis of PACNS. The findings in our case suggest that if PACNS is suspected in a patient with a brain mass, a random skin biopsy prior to brain biopsy should be considered to allow for the avoidance of a more invasive brain biopsy.

IVL is generally considered to proliferate only in vessels. However, some authors have reported that extravasation of malignant lymphocytes can occur to some degree in the late stage of IVL $[1,7]$. To the best of our knowledge, there have been 4 cases of IVL in which postmortem examination revealed a brain mass and 5 cases of tissue-proven IVL in which MRI showed a brain mass (table 1) [8-14]. Our case is notable because it is the first description of DWI of a brain mass in a patient with IVL. DWI is a series of T2-weighted sequences that detect the movement of protons in water. The causes of restricted diffusion on DWI include acute ischemia, cerebral abscesses, diffuse axonal injury, active demyelination, and highly cellular tumors such as lymphoma. DWI change in acute ischemia is commonly known to occur, and marked restricted diffusion in acute ischemia generally remains for 3-5 days. In our case, the restricted diffusion enlarged over 2 weeks, supporting the high cellularity of the mass as the cause of restricted diffusion.

Although there are no pathognomonic presenting symptoms or signs in PCNSL, focal neurological deficits such as personal changes or cerebellar signs are reportedly rather common. Patients with PCNSL may infrequently present with FUO as the initial clinical manifestation [15]. The majority of patients with PCNSL reportedly develop a solitary supratentorial tumor, and the most common sites are the frontal lobe, temporal lobe, parietal lobe, deep nuclei, occipital lobe, cerebellum, or the brain stem. Considering the fact that there are no specific symptoms or signs of PCNSL and that the brain mass noted in our case was a solitary mass located in the basal ganglia, which is a common site of PCNSL, the present case could have been misdiagnosed as PCNSL instead of IVL if the random biopsy had not been performed. The main avenue of treatment for IVL is identical to that for systemic lymphoma. A combination of cyclophosphamide, doxorubicin, vincristine, and prednisone with the recombinant anti-CD20 antibody rituximab (R-CHOP) is the most commonly employed treatment in patients with IVL. On the contrary, CHOP is either ineffective or toxic in the treatment of PCNSL, and methotrexate with brain radiation therapy is usually employed. If the skin biopsy had not been performed, this patient could have been treated by methotrexate, which is not effective against IVL. 


\begin{tabular}{l|l}
\hline Case Rep Neurol 2014;6:101-108 \\
\hline DOI: $10.1159 / 000362121$ & $\begin{array}{l}\text { C 2014 S. Karger AG, Basel } \\
\text { www.karger.com/crn }\end{array}$
\end{tabular}

Oomura et al.: Intravascular Lymphomatosis Mimicking Primary Central Nervous System Lymphoma: A Case Report and Literature Review

\section{Conclusion}

We reported a case of IVL presenting as FUO. MRI showed a brain mass mimicking PCNSL; however, a random skin biopsy confirmed the diagnosis of IVL. Although postmortem examination was not performed, the brain mass noted on MRI was considered to be a collection of malignant lymphomatous cells invading from the vessels. Further accumulation of cases is required to elucidate the full nature of IVL.

\section{References}

1 Wick MR, Mills SE: Intravascular lymphomatosis: clinicopathologic features and differential diagnosis. Semin Diagn Pathol 1991;8:91-101.

-2 DiGiuseppe JA, Nelson WG, Seifter EJ, Boitnott JK, Mann RB: Intravascular lymphomatosis: a clinicopathologic study of 10 cases and assessment of response to chemotherapy. J Clin Oncol 1994;12:2573-2579.

-3 Namekawa M, Nakano I: Diagnosis of intravascular lymphoma: usefulness of random skin biopsies (in Japanese). Brain Nerve 2011;63:451-458.

4 Asada N, Odawara J, Kimura S, Aoki T, Yamakura M, Takeuchi M, Seki R, Tanaka A, Matsue K: Use of random skin biopsy for diagnosis of intravascular large B-cell lymphoma. Mayo Clin Proc 2007;82:1525-1527.

5 Baehring JM, Henchcliffe C, Ledezma CJ, Fulbright R, Hochberg FH: Intravascular lymphoma: magnetic resonance imaging correlates of disease dynamics within the central nervous system. J Neurol Neurosurg Psychiatry 2005;76:540-544.

6 You G, Yan W, Zhang W, Li S, Li G, Jiang T: Isolated angiitis of the central nervous system with tumor-like lesion, mimicking brain malignant glioma: a case report and review of the literature. World J Surg Oncol 2011;9:97-101.

7 Usuda H, Homma K, Nemoto K, Ohnishi Y: Non-Hodgkin's lymphoma with histological features of neoplastic angioendotheliosis. Acta Pathol Jpn 1992;42:272-278.

8 Crosby T: Neoplastic angioendotheliosis (abstr). J Neuropathol Exp Neurol 1976,35:361.

-9 Beal MF, Fisher CM: Neoplastic angioendotheliosis. J Neurol Sci 1982;53:359-375.

10 Ferry JA, Harris NL, Picker LJ, Weinberg DS, Rosales RK, Tapia J, Richardson EP Jr: Intravascular lymphomatosis (malignant angioendotheliomatosis). A B-cell neoplasm expressing surface homing receptors. Mod Pathol 1988;1:444-452.

11 Raroque HG Jr, Mandler RN, Griffey MS, Orrison WW, Kornfeld M: Neoplastic angioendotheliomatosis. Arch Neurol 1990;47:929-930.

12 Massimino M, Giardini R, Cefalo G, et al: Intravascular lymphomatosis (IL) in a child mimicking a posterior fossa tumor. J Neurooncol 2001;51:47-50.

13 Williams RL, Meltzer CC, Smirniotopoulos JG, Fukui MB, Inman M: Cerebral MR imaging in intravascular lymphomatosis. AJNR Am J Neuroradiol 1998;19:427-431.

14 Takeuchi Y, Hashizume Y, Hoshiyama M, Hirose Y, Takahashi A: An autopsy case of intravascular malignant lymphomatosis with intracranial lymphomatous mass lesions (in Japanese). Rinsho Shinkeigaku 1995;35:158-163.

15 Salih SB, Saeed AB, Alzahrani M, Al Qahtani M, Haider A, Palker V: Primary CNS lymphoma presenting as fever of unknown origin. J Neurooncol 2009;93:401-404. 
Oomura et al.: Intravascular Lymphomatosis Mimicking Primary Central Nervous System Lymphoma: A Case Report and Literature Review

Table 1. Summary of reported cases of IVL with brain mass

\begin{tabular}{lllll}
\hline Reference & $\begin{array}{l}\text { Age/ } \\
\text { sex }\end{array}$ & Presentation & Mass lesion & Modality \\
\hline Crosby [8] & 60/? & Dementia and fever & Occipital white matter & Autopsy \\
\hline Beal and Fisher [9] & $57 / \mathrm{M}$ & $\begin{array}{l}\text { Numbness and slurred } \\
\text { speech }\end{array}$ & Bilateral globus pallidus & Autopsy \\
\hline Ferry et al. [10] & 58/M & TIA & Bilateral thalamus & Autopsy \\
\hline Raroque et al. [11] & 60/M & Seizure and paraparesis & Bilateral frontal lobe & MRI w/o DWI \\
\hline Takeuchi et al. [14] & 50/M & Gait disturbance & $\begin{array}{l}\text { Bilateral caudate nucleus and } \\
\text { RRI and autopsy }\end{array}$ \\
\hline Williams et al. [13] & 32/M & Weakness and confusion & $\begin{array}{l}\text { R frontal lobe and L parietal } \\
\text { lobe }\end{array}$ & MRI w/o DWI \\
\hline Williams et al. [13] & 66/M & Confusion & L parietooccipital lobe & MRI w/o DWI \\
\hline Williams et al. [13] & 64/M & Seizure & R temporal lobe & MRI w/o DWI \\
\hline Massimino et al. [12] & 13/F & Vertigo & Cerebellar vermis & MRI w/o DWI \\
\hline Our case & 75/F & FU0 & R basal ganglia and thalamus & MRI with DWI \\
\hline
\end{tabular}

TIA = Transient ischemic attack; w/o = without. 
Oomura et al.: Intravascular Lymphomatosis Mimicking Primary Central Nervous System Lymphoma: A Case Report and Literature Review

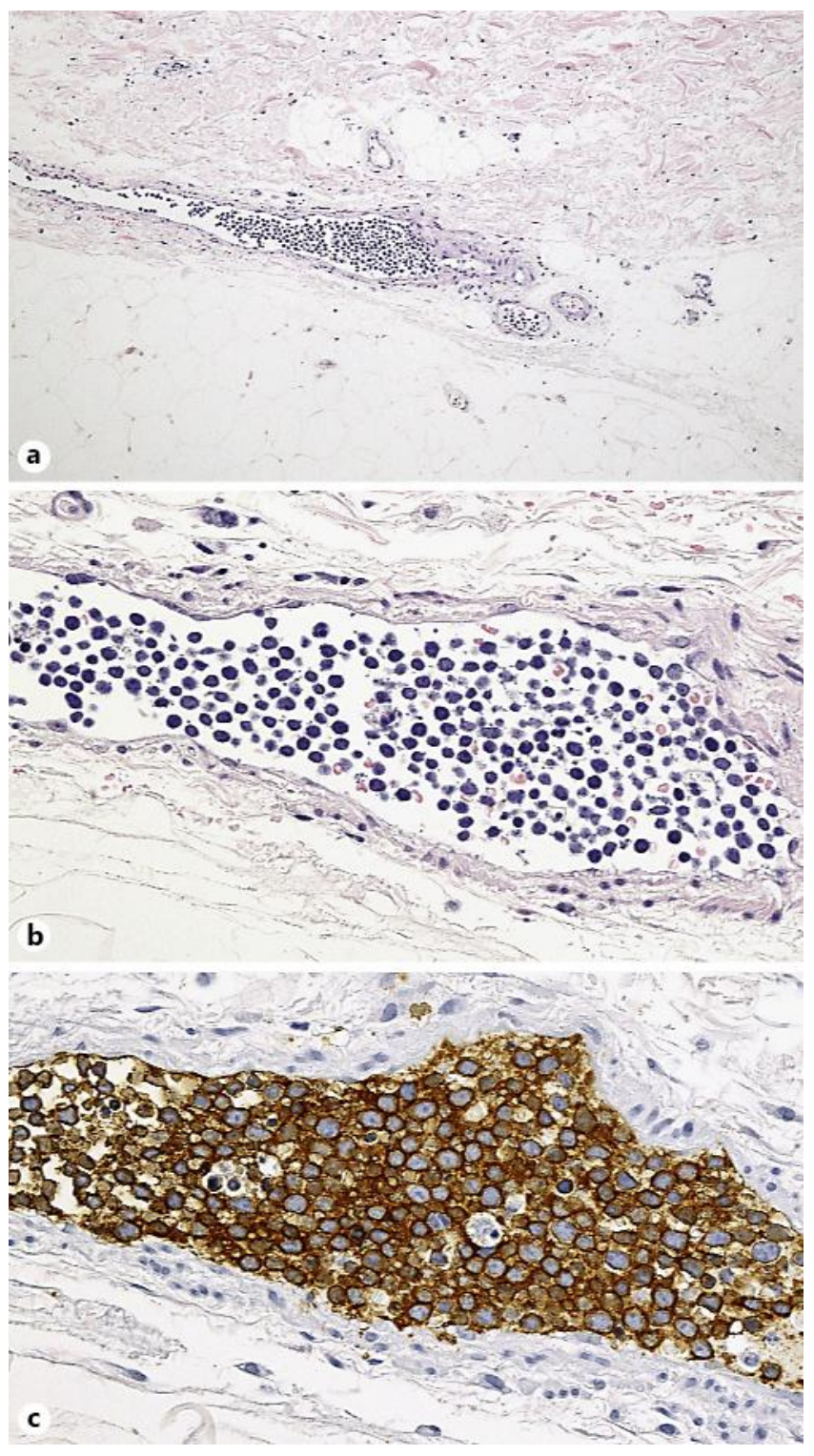

Fig. 1. Skin biopsy revealed large malignant lymphoid cells with eosinophilic nucleoli in the vessels of the subcutaneous tissue (HE. $\mathbf{a} \times 100 ; \mathbf{b} \times 400)$. The tumor cells in the vessels are highlighted by immunohistochemical staining for $\mathrm{CD} 20(\mathrm{c} \times 400)$. 


\section{Case Reports in Neurology}
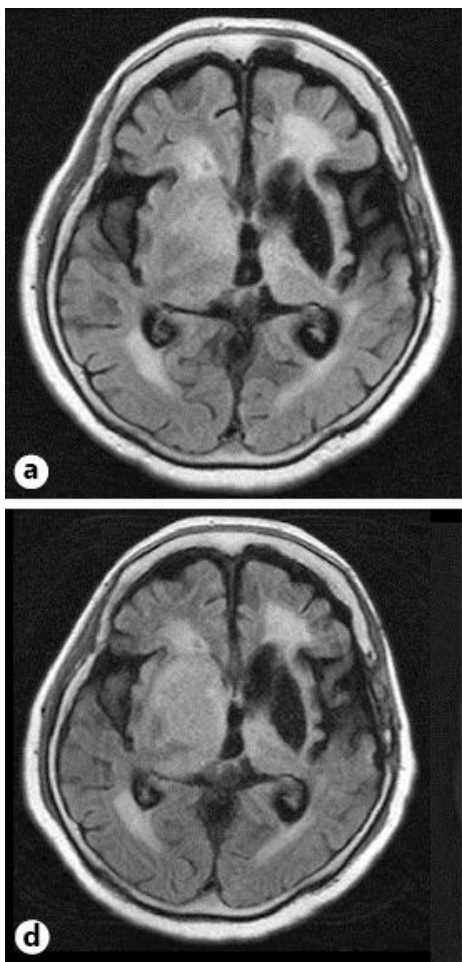

Case Rep Neurol 2014;6:101-108

DOI: 10.1159/000362121

Oomura et al.: Intravascular Lymphomatosis Mimicking Primary Central Nervous
System Lymphoma: A Case Report and Literature Review
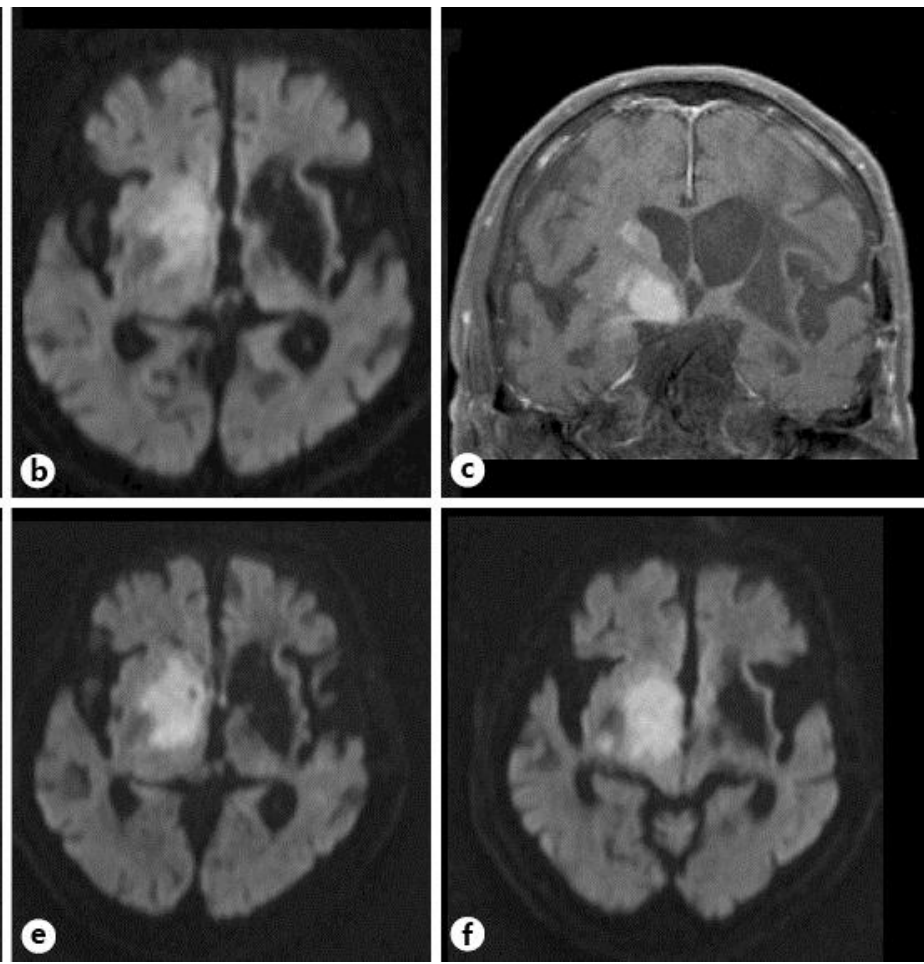

Fig. 2. Brain MRI in the 7th (a-c) and 9th (d-f) week after admission. FLAIR and DWI show a mass involving the right basal ganglia and thalamus $(\mathbf{a}, \mathbf{b})$. The mass was clearly enhanced (c). Two weeks later, the mass had enlarged $(\mathbf{d}-\mathbf{f})$. 